\title{
Marcadores de aterosclerose subclínica no diabetes
}

\author{
Alessandra S. M. Matheus, ${ }^{1 *}$ Cátia C. S. S. V. Palma, ${ }^{1}$ Carlos Roberto M. de Andrade Júnior ${ }^{1}$
}

\section{Resumo}

Este capítulo tem como objetivo avaliar as técnicas de detecção de aterosclerose subclínica para melhorar a estratificação do risco cardiovascular em pacientes diabéticos assintomáticos. A função endotelial, a reatividade microvascular, a medida da espessura íntima-média (EIM carotídea) e a medida da calcificação coronariana são alguns dos marcadores que já podem estar alterados mesmo em pacientes com diagnóstico recente de diabetes mellitus. Pelo fato de estarem presentes desde o início da lesão aterosclerótica, podem ser considerados marcadores precoces de aterosclerose subclínica. A estratificação do risco cardiovascular com esses marcadores precoces visam melhorar a capacidade de avaliarmos o "verdadeiro" risco. Os pacientes com maior risco podem se beneficiar de estratégias clínicas mais agressivas e mais precoces do que os pacientes com baixo risco. Algumas das técnicas que são utilizadas para detecção precoce de aterosclerose subclínica: fluxometria por laser Doppler, tonometria da artéria periférica (Endo-PAT), escore de cálcio (EC) e avaliação da espessura íntima-média (EIM) são descritas. Essas técnicas podem ser utilizadas para melhorar a avaliação do risco cardiovascular em pacientes assintomáticos. Quando utilizadas em conjunto com os escores clínicos já estabelecidos, como o de Framingham e/ou do UKPDS, aumentam ainda mais a acurácia da determinação do risco. Outras técnicas podem ser utilizadas, porém com alto custo e baixa reprodutibilidade. O rastreio precoce da doença aterosclerótica subclínica em pacientes assintomáticos assume relevante importância diante da morbimortalidade da doença cardiovascular e suas complicações em pacientes diabéticos. Apesar da extrema importância, não há ainda consenso nem protocolos estabelecidos sobre quais exames e técnicas devem ser preconizadas nestes pacientes.

Descritores: Risco; Doenças cardiovasculares; Aterosclerose; Diabetes mellitus.

\section{Abstract}

\section{Subclinical atherosclerosis markers in diabetes}

The objective of this chapter is to evaluate the current methods to detect subclinical atherosclerosis in patients with diabetes that may improve the cardiovascular risk stratification in these patients. Some of these methods that analyze the endothelial function such as microvascular reactivity, measurement of the carotid intima-media thickness (IMT), and the measurement of coronary calcification are some of the markers that may be altered even in patients with recent diagnoses of Diabetes. Indeed, these alterations may be present since the start of the atherosclerotic lesion and can be considered as precocious markers of subclinical atherosclerosis. The
1. Serviço de Diabetes. Hospital Universitário Pedro Ernesto. Universidade do Estado do Rio de Janeiro. Rio de Janeiro, RJ, Brasil

\author{
*Endereço para correspondência: \\ HUPE, UERJ \\ Boulevard 28 de Setembro, 77 \\ Rio de Janeiro, RJ, Brasil. CEP: 20551-030. \\ E-mail: alessandramatheus79@yahoo.com
}

Revista HUPE, Rio de Janeiro, 2015;14(4):59-63

doi: 10.12957/rhupe.2015.20059

Recebido em 28/11/2014. Aprovado em 16/01/2015.

stratification of cardiovascular risk with these early markers may improve the ability to assess the "real" cardiovascular risk. Patients at higher risk may benefit from earlier and more aggressive clinical strategies. In this review we will describe some of the techniques that are used for the early detection of subclinical atherosclerosis: laser Doppler flowmeter, peripheral artery tonometry (Endo-PAT), calcium score (CS) and assessment of the arterial intima-media thickness (IMT) are described. These techniques can be used to improve the assessment of cardiovascular risk in asymptomatic patients. When used in conjunction with established clinical scores such as the Framingham score and the UKPDS engine, there may be further improvement in the stratification of the cardiovascular risk. Other techniques can be used but with high cost and low reproducibility. The early detection of subclinical atherosclerosis in asymptomatic patients assumes great importance on morbidity and mortality of cardiovascular disease in patients with diabetes. Even though extremely important, there is no consensus or established protocols on which tests and techniques should be recommended in these patients.

Keywords: Risk; Cardiovascular diseases; Atherosclerosis; Diabetes mellitus.

\section{Resumen}

\section{Marcadores de aterosclerosis subclínica en la dia- betes}

Este capítulo tiene como objetivo evaluar las técnicas de detección de aterosclerosis subclínica para mejorar la estratificación del riesgo cardiovascular en pacientes diabéticos asintomáticos. La función endotelial, la reactividad microvascular, la medición del grosor íntima-media (GIM carotídeo) y el grado de calcificación coronaria, son algunos de los maracadores que ya pueden estar alterados incluso en pacientes con diagnóstico 
reciente de diabetes mellitus. Debido a que están presentes desde el inicio de la lesión aterosclerótica, pueden ser considerados como marcadores tempranos de aterosclerosis subclínica. La estratificación del riesgo cardiovascular con estos marcadores tempranos está destinada a mejorar la capacidad de evaluar el "verdadero" riesgo. Los pacientes con mayor riesgo pueden beneficiarse de estrategias clínicas más agresivas y más precoces que los pacientes con bajo riesgo. Algunas de las técnicas que se utilizan para la detección precoz de la aterosclerosis subclínica: flujometría por laser Doppler, tonometría arterial periférica (Endo-PAT), score de calcio (SC) y la evaluación del grosor íntima-media (GIM) son descritas. Estas técnicas se pueden utilizar para mejorar la evaluación del riesgo cardiovascular

\section{Introdução}

A hiperglicemia e outros fatores de risco cardiovascular (RCV) atuam de forma sinérgica nos pacientes com diabetes levando às complicações macrovasculares. Assim, a morbidade e a mortalidade por doença cardiovascular (DCV) podem estar aumentadas em cerca de três a cinco vezes em pacientes com diabetes mellitus tipo 2 (DM2) e até dez vezes em pacientes com diabetes mellitus tipo 1(DM1). No início desse processo, a hiperglicemia e os outros fatores de RCV levariam à disfunção endotelial, que progressivamente evoluiria para um processo de aterosclerose subclínica e posteriormente para eventos macrovasculares clínicos.

Com isso, torna-se necessária a identificação desse processo de aterosclerose ainda numa fase pré-clínica e assim a identificação daqueles pacientes que se beneficiariam de um tratamento mais intensivo e precoce dos seus fatores de RCV.

Nos últimos anos, diferentes métodos para a identificação da disfunção endotelial e do processo de aterosclerose estão em desenvolvimento ainda na fase pré-clínica, dentre os quais podemos citar os estudos dinâmicos da microcirculação, o escore de cálcio e a espessura da camada íntima-média da artéria carótida comum.

O objetivo deste capítulo é descrever técnicas atuais de detecção do processo de aterosclerose subclínica e de estratificação do RCV em pacientes com diabetes.

\section{Avaliação da função endotelial como marcador de aterosclerose subclínica}

A disfunção endotelial aparece como um denominador comum na fisiopatologia das complicações crônicas no diabetes e está intimamente relacionada com o risco cardiovascular. ${ }^{1}$ Pacientes com diabetes e disfunção endotelial apresentam maiores taxas de en pacientes asintomáticos. Cuando se utilizan junto con las puntuaciones clínicas ya establecidas como el Framingham y / o el UKPDS, aumentan aún más la precisión de la determinación del riesgo. Otras técnicas se pueden utilizar, pero con alto costo y baja reproducibilidad. La detección precoz de la aterosclerosis subclínica en pacientes asintomáticos asume gran importancia frente a la morbimortalidad de las enfermedades cardiovasculares y sus complicaciones en los pacientes diabéticos. A pesar de la mayor importancia, todavía no hay consenso o protocolos establecidos sobre qué exámenes y técnicas deben recomendarse en estos pacientes.

Palabras clave: Riesgo; Enfermedades cardiovasculares; Aterosclerosis; Diabetes mellitus.

eventos cardiovasculares quando comparados com aqueles com função endotelial preservada e a vasodilatação mediada pelo fluxo que se encontra reduzida nos diabéticos mostrou-se ser um preditor independente de reestenose após intervenções coronarianas. ${ }^{1,2} \mathrm{~A}$ função endotelial e a reatividade microvascular encontram-se alteradas desde as fases precoces no diabetes, ${ }^{3}$ e pelo fato de estarem presentes no início da lesão aterosclerótica podem ser consideradas marcadores precoces de aterosclerose subclínica.

No diabetes, a disfunção endotelial pode ser caracterizada pela redução na vasodilatação dependente do endotélio, ${ }^{4-6}$ e "ativação endotelial" estando associada com um ambiente proliferativo pró-aterogênico, pró-inflamatório e pró-coagulante que participa da formação da placa de ateroma e demais complicações ateroscleróticas?

Devido à crescente relevância da predição do risco cardiovascular a longo prazo, vem aumentando o interesse nas técnicas não invasivas de avaliação da função endotelial e dos biomarcadores de disfunção endotelial. ${ }^{8,9}$

O método padrão-ouro para a avaliação do fluxo sanguíneo in vivo é a cateterização coronariana por angiografia e por doppler, no entanto, devido ao caráter altamente invasivo e pelo alto custo, está restrito a poucos centros de pesquisa. ${ }^{10}$ De maneira geral, o custo, a subjetividade, a alta variabilidade e a ausência de validação têm reduzido a confiabilidade de outros métodos funcionais, como o ultrassom e a pletismografia por oclusão venosa. Atualmente, as sofisticadas técnicas de imagens para o estudo da função endotelial, tais como a tomografia com emissão de pósitrons em três dimensões, a ressonância magnética e a ecografia contrastada, apresentam como principal limitação à sua aplicabilidade os elevados custos envolvidos. ${ }^{11}$ 
A fluxometria por laser doppler, a tonometria da artéria periférica (Endo-PAT) e o laser speckle imagem de contraste (LSCI) despontam como técnicas não invasivas promissoras por apresentarem uma alta reprodutibilidade ( $\mathrm{LSCI}^{12}$ e Endo-PAT ${ }^{13}$ ), correlação com a função coronariana ${ }^{2,14}$ (Endo-PAT) e serem de fácil aplicabilidade e independentes do operador. ${ }^{15}$ No entanto, por serem técnicas recentes, ainda carecem de validação e padronização dos estudos em diabetes.

Os biomarcadores de disfunção endotelial desempenham relevante papel no diagnóstico e prognóstico, principalmente para complicações microvasculares e sua avaliação também pode ser útil no estudo da fisiopatologia da aterosclerose precoce no diabetes. ${ }^{16}$ Dentre os biomarcadores, as moléculas de adesão apresentam um papel importante nos primeiros estágios da aterosclerose. Níveis elevados de VCAM-1 encontrados em pacientes com DM1 jovens são correlacionados com descompensação metabólica e história familiar de doenças cardiovasculares. A E-selectina representa um marcador específico de ativação endotelial e mediador angiogênico por surgir como coadjuvante na patogênese das complicações microvasculares encontradas no diabetes ${ }^{17}$ e níveis séricos elevados encontram-se associados à disfunção endotelial. A Interleucina-6 é uma citocina pró-inflamatória que está envolvida na angiogênese, aterosclerose e lesão endotelial. ${ }^{17}$

Apesar das evidências demonstrarem um bom valor preditivo diagnóstico para eventos cardiovasculares, a avaliação da função endotelial é considerada classe III e ainda não é preconizada pelos Consensos Americano e Europeu de Cardiologia. O estudo da função endotelial por técnicas não invasivas e mais recentes recebeu uma classificação inferior ao Escore Coronariano de Cálcio e ao espessamento da íntimamédia carotídea por não apresentar protocolos padronizados e carecer de estudos validados, com a exceção da técnica do Endo-PAT.

\section{Avaliação do escore de cálcio}

Na tentativa de se identificar dentre os pacientes assintomáticos aqueles que apresentam maior risco de eventos cardiovasculares, várias estratégias de detecção estão sendo desenvolvidas. ${ }^{18}$ A utilização de escores clínicos (tais como Framingham e UKPDS) de forma isolada pode ser uma limitação na estratificação do risco cardiovascular (RCV) em uma grande parte da população, principalmente em mulheres e em grupos mais jovens. ${ }^{19}$

O escore de cálcio coronariano (EC) pode ser con- siderado como uma ferramenta complementar na estratificação do RCV de pacientes assintomáticos. ${ }^{20} \mathrm{~A}$ determinação do EC coronariano se dá pela obtenção de imagens não contrastadas de cortes axiais com 3 mm de espessura cobrindo toda a extensão do coração. As imagens são captadas de forma sincronizada ao sinal do eletrocardiograma, podendo ser de forma prospectiva e retrospectiva. Devido a uma maior quantidade de radiação na forma retrospectiva, os protocolos utilizando a forma prospectiva são mais utilizados. ${ }^{19}$ A definição da calcificação é uma lesão hiperatenuante com intensidade de sinal acima de 130 unidades Hounsfield (HU) e área >= 3 pixels adjacentes (de pelo menos $1 \mathrm{~mm}$ ). O escore de Agatston é a soma ponderada das densidades acima de $130 \mathrm{HU}$, sendo este o mais utilizado quando se descreve a distribuição da calcificação coronariana de acordo com a idade, etnia e sexo dos pacientes. ${ }^{21}$ Métodos que utilizam a determinação do volume ou massa de cálcio também podem ser utilizados. A classificação do EC pode ser feita de duas formas: por pontos de cortes fixos com valores já pré-estabelecidos ou por pontos de corte ajustados, em que os valores são em percentis de distribuição dos valores da população geral. Ambas as formas descrevem informações prognósticas valiosas, principalmente na estratificação de risco de pacientes assintomáticos. ${ }^{19}$

O EC não deve ser usado como ferramenta diagnóstica para doença arterial coronariana obstrutiva significativa em pacientes com sintomatologia coronariana. A calcificação coronariana demonstra a presença de placas ateroscleróticas nas artérias coronárias e isso corresponde a cerca de $20 \%$ do volume total das placas e se correlaciona positivamente com a maior chance de evento cardiovascular no futuro. Em um estudo observacional realizado por Kalia e colaboradores, nos pacientes que apresentaram uma maior calcificação pelo EC houve um aumento de 46\% no uso de medicações hipolipemiantes. ${ }^{22}$

De acordo com o posicionamento da Sociedade Brasileira de Diabetes em relação ao diabetes e à doença cardiovascular, em pacientes com risco intermediário calculado através da calculadora do UKPDS ${ }^{23}$ a realização do EC, quando disponível, está indicada como ferramenta de reclassificação do risco para baixo ou alto, com nível A de evidência. ${ }^{20}$ A utilização do EC deve ser adicional aos métodos de estratificação do risco pelo escore de Framingham, dosagem de proteína $\mathrm{C}$ reativa, por exemplo, na predição de eventos cardiovasculares. 


\section{Artigo de revisão}

\section{Avaliação do espessamento da íntima- média carotídea}

Alguns autores demonstraram que a medida da espessura da camada que engloba a íntima e a média (EIM) da artéria carótida comum através de ultrassonografia está relacionada à disfunção endotelial e consequentemente ao risco cardiovascular (RCV) aumentado em pacientes com ou sem diabetes, assim, consolidando-se como um marcador de aterosclerose subclínica. ${ }^{2428}$ Acredita-se que exista uma correlação linear entre o aumento do risco de morte cardiovascular total e o aumento da EIM carotídea, sendo este capaz de diferenciar aqueles pacientes com RCV elevado daqueles com baixo RCV..$^{29}$

A medida da EIM carotídea, segundo as recomendações do consenso da American Society of Echocardiography (ASE), é um procedimento não invasivo, relativamente simples e rápido..$^{30}$ Os valores de normalidades foram definidos de acordo com o sexo, a idade e a etnia do indivíduo avaliado. A medida da EIM no terço distal da artéria carótida comum parece se relacionar melhor com o RCV quando comparado à medida EIM em outro ponto da artéria carótida. ${ }^{31}$

Múltiplos fatores parecem estar associados à EIM. Em pacientes com DM1, a história familiar de DM2 parece ser fator determinante, estando associada à maior prevalência de obesidade e critérios para síndrome metabólica. ${ }^{32} \mathrm{Um}$ estudo publicado pelo nosso grupo, com pacientes com DM1, sugeriu que a presença de história familiar de DM2 em parentes de primeiro grau estaria associada a um aumento de cerca de 4,4\% na EIM. ${ }^{33}$ Nesse mesmo estudo, os autores sugeriram também que o sexo feminino estaria associado ao aumento de 2,9\%, cada ano de duração do DM1 ao aumento de $0,4 \%$, cada ano de idade ao aumento de $0,6 \%$ e cada 1 ponto de aumento do IMC a um aumento de $0,5 \%$ da EIM. Em pacientes com DM2, a hipertrigliceridemia, principalmente pós-prandial, parece ter papel relevante do aumento da EIM. ${ }^{34}$ Além disso, um autor sugeriu que a redução de $10 \%$ do colesterol LDL com o uso de estatinas poderia reduzir a EIM carotídea em 0,75\% ao ano..$^{35}$ Assim, a determinação dos fatores associados ao aumento da EIM carotídea é complexa e inúmeros outros fatores, além dos descritos acima, parecem estar associados, incluindo o maior tempo de doença, menor idade ao diagnóstico, sexo do paciente, maiores índice de massa corpórea (IMC) e de relação cintura-quadril, etnia do paciente, maiores pressão arterial sistólica e diastólica, maior dose diária de insulina, terapia de reposição hormonal nas mulheres, tabagismo, presença de retinopatia e de nefropatia, HDL baixo e elevação de marcadores de inflamação sistêmica como a proteína C reativa (PCR). ${ }^{36}$

A utilização da EIM e da idade vascular poderiam melhorar a capacidade de avaliarmos o verdadeiro RCV de pacientes com diabetes, uma vez que a idade vascular estimada pela EIM consideraria a interação de fatores protetores e potencializadores do RCV, conhecidos ou não. Diferentemente, ao utilizarmos ferramentas como o escore de Framingham, estamos avaliando cada fator de risco separadamente, sem considerarmos a interação entre eles. ${ }^{37}$

\section{Conclusões}

A análise da função endotelial e da reatividade microvascular, que estão alteradas desde as fases mais precoces no diabetes e presentes na lesão aterosclerótica, podem ser consideradas ferramentas para a determinação do risco cardiovascular em pacientes assintomáticos. Entretanto, as técnicas consideradas padrão-ouro são invasivas e de alto custo. As novas técnicas para análise da função endotelial, com exceção do Endo-PAT, ainda não possuem protocolos padronizados e carecem de estudos validados, porém são técnicas promissoras, com vários estudos em andamento.

O escore de cálcio (EC) deve ser utilizado como uma ferramenta complementar na estratificação de risco cardiovascular no paciente assintomático. A utilização deste escore em conjunto com os escores clínicos já estabelecidos (como o UKPDS e o Framingham) pode reclassificar os pacientes com risco intermediário em alto ou baixo risco. $O$ alto custo da técnica pode limitar a sua utilização em larga escala.

A análise da EIM se correlaciona de forma linear à disfunção endotelial e ao risco cardiovascular, sendo uma técnica simples e não invasiva. A utilização da "idade vascular" (correspondente ao p50 da medida da EIM carotídea do paciente avaliado) pode ser um melhor preditor do RCV dos pacientes assintomáticos com ou sem diabetes.

No entanto, ainda não há consenso nestas recomendações e, muito menos, não existem protocolos estabelecendo quais exames de rastreio devem ser preconizados no diabético assintomático.

\section{Referências}

1. Kolluru GK, Bir SC, Kevil CG. Endothelial dysfunction and diabetes: effects on angiogenesis, vascular remodeling, and wound healing. Int J Vasc Med. 2012;2012:918267.

2. Flammer AJ, Anderson T, Celermajer DS, et al. The Assessment of Endothelial Function: From Research Into Clinical Practice. 
Circulation. 2012;126(6):753-67

3. Basha B, Samuel SM, Triggle CR, et al. Endothelial Dysfunction in Diabetes Mellitus: Possible Involvement of Endoplasmic Reticulum Stress? Exp Diabetes Res. 2012;2012:1-14.

4. Skrha J, Prazny M, Haas T, et al. Comparison of laser-Doppler flowmetry with biochemical indicators of endothelial dysfunction related to early microangiopathy in Type 1 diabetic patients. J Diabetes Complications. 2001 Sep-Oct;15(5):234-40.

5. Gomes MB, Matheus AS, Tibirica E. Evaluation of microvascular endothelial function in patients with type 1 diabetes using laser-Doppler perfusion monitoring: which method to choose? Microvasc Res. 2008 Aug;76(2):132-3.

6. Matheus AS, Tibirica E, da Silva PB, et al. Uric acid levels are associated with microvascular endothelial dysfunction in patients with Type 1 diabetes. Diabet Med. 2011 Oct;28(10):118893.

7. Xu J, Zou MH. Molecular Insights and Therapeutic Targets for Diabetic Endothelial Dysfunction. Circulation. 2009;120(13):1266-86.

8. Cohn JN, Quyyumi AA, Hollenberg NK, et al. Surrogate markers for cardiovascular disease: functional markers. Circulation. 2004 Jun 29;109(25 Suppl 1):IV31-46.

9. Vita JA, Keaney JF Jr. Endothelial function: a barometer for cardiovascular risk? Circulation. 2002 Aug 6;106(6):640-2.

10. Bonetti PO, Lerman LO, Lerman A. Endotelial dysfunction: A marker of atherosclerotic risk. Arterioscler Thromb Vasc Biol. 2003;23:168-75.

11. Struijker-Boudier HAJ, Rosei AE, Bruneval P, et al. Evaluation of the microcirculation and hypertension and cardiovascular disease. Eur Heart J. 2007;28(23):2834-40.

12. Roustit M, Millet C, Blaise S, et al. Excellent reproducibility of laser speckle contrast imaging to assess skin microvascular reactivity. Microvasc Res. 2010 Dec;80(3):505-11.

13. Reisner Y, Lusky R, Shay-El Y, et al. Reproducibility of endothelial function and arterial stiffness assessed using finger peripheral arterial tonometry. Eur Heart J. 2007;28(Supplement):484.

14. Bonetti PO, Pumper GM, Higano ST, et al. Noninvasive identification of patients with early coronary atherosclerosis by assessment of digital reactive hyperemia. J Am Coll Cardiol. 2004 Dec 7;44(11):2137-41.

15. Turner J, Belch JJ, Khan F. Current concepts in assessment of microvascular endothelial function using laser Doppler imaging and iontophoresis. Trends Cardiovasc Med. 2008;18:109-16.

16. Lopes-Virella MF, Carter RE, et al. Risk factors related to inflammation and endothelial dysfunction in the DCCT/EDIC cohort and their relationship with nephropathy and macrovascular complications. Diabetes Care. 2008 Oct;31(10):2006-12.

17. Kuryliszyn A, Dubicki A, Zarzycki W, et al. Microvascular abnormalities in capillaroscopy correlate with higher serum IL-18 and $\mathrm{sE}$-selectin levels in patients with type 1 diabetes complicated by microangiopathy. Folia Histochem Cytobiol. 2011;49(1):10410.

18. Almeida FK, Esteves JF, Gross JL, et al. Severe forms of retinopathy predict the presence of subclinical atherosclerosis in type 1 diabetes subjects. Arq Bras Cardiol. 2011 Oct;97(4):346-9.

19. Azevedo CF, Rochitte CE, Lima JA. Coronary artery calcium score and coronary computed tomographic angiography for cardiovascular risk stratification. Arq Bras Cardiol. 2012 Jun;98(6):559-68.

20. Gualandro DM, Malerbi DA, Moriguchi E, et al. Diabetes and cardiovascular disease: from evidence to clinical practice - position statement 2014 of Brazilian Diabetes Society. 2014;6:58.
21. Ahmed W, de Graaf MA, Broersen A, et al. Automatic detection and quantification of the Agatston coronary artery calcium score on contrast computed tomography angiography. Int J Cardiovasc Imaging. 2014 Aug 27.

22. Kalia NK, Miller LG, Nasir K, et al. Visualizing coronary calcium is associated with improvements in adherence to statin therapy. Atherosclerosis. 2006 Apr;185(2):394-9.

23. Chamnan P, Simmons RK, Sharp SJ, et al. Cardiovascular risk assessment scores for people with diabetes: a systematic review. Diabetologia. 2009 Oct;52(10):2001-14.

24. Frost $D$, Beischer W. Progression of the carotid artery intima-media thickness in young patients with type 1 diabetes. Diabetes Care. 2003 Feb;26(2):545.

25. Holman RR, Paul SK, Bethel MA, et al. 10-year follow-up of intensive glucose control in type 2 diabetes. N Engl J Med. 2008 Oct 9;359(15):1577-89.

26. Bernard S, Serusclat A, Targe F, et al. Incremental predictive value of carotid ultrasonography in the assessment of coronary risk in a cohort of asymptomatic type 2 diabetic subjects. Diabetes Care. 2005 May;28(5):1158-62.

27. Temelkova-Kurktschiev TS, Koehler C, Leonhardt W, et al. Increased intimal-medial thickness in newly detected type 2 diabetes: risk factors. Diabetes Care. 1999 Feb;22(2):333-8.

28. Halcox JP, Donald AE, Ellins E, et al. Endothelial function predicts progression of carotid intima-media thickness. Circulation. 2009 Feb 24;119(7):1005-12.

29. Ashrafian H, Lim TK, Senior R. Carotid ultrasound imaging: an effective technique for detecting early atherosclerosis-vascular screening for cardiovascular disease has come of age. J Am Soc Echocardiogr. 2007 Jan;20(1):1-3.

30. Simons PC, Algra A, Bots ML, et al. Common carotid intima-media thickness and arterial stiffness: indicators of cardiovascular risk in high-risk patients. The SMART Study (Second Manifestations of ARTerial disease). Circulation. 1999 Aug 31;100(9):951-7.

31. Chambless LE, Heiss G, Folsom AR, et al. Association of coronary heart disease incidence with carotid arterial wall thickness and major risk factors: the Atherosclerosis Risk in Communities (ARIC) Study, 1987-1993. Am J Epidemiol. 1997 Sep 15;146(6):483-94.

32. Orchard TJ, Costacou T, Kretowski A, et al. Type 1 diabetes and coronary artery disease. Diabetes Care. 2006 Nov;29(11):2528-38.

33. Andrade CRM, Silva ELC, Matta MFB, et al. Influence of a family history of type 2 diabetes, demographic and clinical data on carotid intima-media thickness in patients with type 1 diabetes: a cross-sectional study. Cardiovasc Diabetol. 2014;13:87-95.

34. Teno S, Uto Y, Nagashima H, et al. Association of postprandial hypertriglyceridemia and carotid intima-media thickness in patients with type 2 diabetes. Diabetes Care. 2000 Sep;23(9):1401-6.

35. Amarenco P, Donnan GA. Statins in stroke prevention and carotid atherosclerosis: systematic review and up-to-date meta-analysis. Stroke. 2004 Dec;35(12):2902-9.

36. Volzke H, Robinson DM, Schminke U, et al. Thyroid function and carotid wall thickness. J Clin Endocrinol Metab. 2004 May;89(5):2145-9.

37. Junyent M, Zambon D, Gilabert R, et al. Carotid atherosclerosis and vascular age in the assessment of coronary heart disease risk beyond the Framingham Risk Score. Atherosclerosis. 2008 Feb;196(2):803-9. 